\title{
Profiling of Ionospheric Electron Density Based on FormoSat-3/COSMIC Data: Results from the Intense Observation Period Experiment
}

\author{
Lung-Chih Tsai ${ }^{1,3,}{ }^{*}$, Chao-Han $\mathrm{Liu}^{2}$, and Tung-Yuan Hsiao ${ }^{3}$ \\ ${ }^{1}$ Center for Space and Remote Sensing Research, National Central University, Chung-Li, Taiwan, ROC \\ ${ }^{2}$ Academia Sinica, Taipei, Taiwan, ROC \\ ${ }^{3}$ Graduate Institute of Space Science, National Central University, Chung-Li, Taiwan, ROC
}

Received 1 May 2007, accepted 19 December 2007

\begin{abstract}
Multi-channel Global Position System (GPS) carrier phase signals received by the FormoSat-3 (FS3)/COSMIC program's six low Earth orbiting (LEO) satellites were used to undertake active limb sounding of the Earth's atmosphere and ionosphere via radio occultation (RO). In this study, the effects of large-scale horizontal gradients and/or inhomogeneous ionospheric electron densities $\left(n_{e}\right)$ were considered by developing an iterative scheme to determine "compensated" total electron content (TEC) values through nearby occultation observations. Using an iterative Abel inversion through "compensated" TEC values, more than one-hundred and eighty thousand vertical ionospheric $n_{e}$ profiles were collected during the intense observation period (IOP) of 20 June - 27 September (day number 171 to 270) 2006. Coincidences of GPS RO observations to ionospheric $n_{e}$ ionosonde data from forty-nine worldwide stations have been examined. It is found that the iterative scheme gives improved retrievals over the standard Abel inversion. From several thousand matches within the IOP experiment, the root mean square $(\mathrm{rms})$ foF 2 differences between the ionosonde measurements and the FS3/COSMIC retrievals are improved from 1.67 to $1.07 \mathrm{MHz}$. The results also show improvement in the overestimates (underestimates) on low (high) retrieved $f_{0} F 2 \mathrm{~s}$, while the obtained $h m F 2$ s give better agreement with ionosonde observations.
\end{abstract}

Key words: FormoSat-3/COSMIC, Ionosphere, Radio occultation, Electron density, Abel inversion, Total electron content (TEC)

Citation: Tsai, L. C., C. H. Liu, and T. Y. Hsiao, 2009: Profiling of ionospheric electron density based on FormoSat-3/COSMIC data: Results from the intense observation period experiment. Terr. Atmos. Ocean. Sci., 20, 181-191, doi: 10.3319/TAO.2007.12.19.01(F3C)

\section{INTRODUCTION}

Radio occultations (RO) have long been used in the remote sensing of planetary atmospheres (Fjeldbo et al. 1969). Receiving multi-channel Global Positioning System (GPS) carrier phase signals from a low Earth orbiting (LEO) satellite and probing Earth's atmosphere and ionosphere via occultation was proposed by the Jet Propulsion Laboratory in 1988. However, it was first implemented by the University Corporation for Atmospheric Research (UCAR) (Zou et al. 1995; Ware et al. 1996; Kursinski et al. 1997; Rocken et al. 1997) as the GPS/Meteorology (GPS/MET) program. The main objectives of this GPS-LEO occultation mission were numerical weather prediction and long-term monitoring of Earth's climate. After the GPS/MET mission, further mis-

\footnotetext{
* Corresponding author

E-mail:lctsai@csrsr.ncu.edu.tw
}

sions flown with GPS occultation receivers onboard include the Danish Ørsted, the German CHAMP, the Argentinean SAC-C, the American-German GRACE, the South African SUNSAT, and the Ionosphere Occultation Experiment (IOX) onboard the PICOSAT satellite.

The Taiwan FS3/Constellation Observing System for Meteorology, Ionosphere, and Climate (COSMIC) satellites were successfully launched on 15 April 2006. In relation to the GPS/MET, CHAMP, and SAC-C missions, the FS3/ COSMIC project's goal was to place six micro-satellites into six different orbits at $\sim 700-800 \mathrm{~km}$ altitudes. Each microsatellite is equipped with two high-gain occultation antennas to track rising and setting GPS satellites. The FS3/COSMIC RO measurements will cover the entire global atmosphere and ionosphere, providing over 2500 soundings per day. All six spacecrafts (FM1 to FM6) were integrated and launched 
together; whereupon, each spacecraft was separated and then transferred orbits from 500 to $800 \mathrm{~km}$. During day number 171 to 270 in 2006, the FM2 spacecraft was in the $800 \mathrm{~km}$ mission orbit, while the other five spacecraft were waiting for orbit rising in adjacent parking orbits of $\sim 500 \mathrm{~km}$ altitude. The IOP experiment along the FM1, FM3 - FM6 parking orbit can thus provide quintuple data and more dense observations than a single spacecraft.

The operational ionospheric processing for FS3/COSMIC radio occultation data follows the scheme used for the GPS/MET data (Schreiner et al. 1999). These retrieval processes generally assume spherical symmetry of electron density $\left(n_{e}\right)$ at the occultation location and the Abel integral transform is used to invert total electron contact (TEC) data to one-dimensional $n_{e}$ as a function of tangent point altitude (Hajj and Romans 1998; Hocke and Igarashi 2002). There are several works which examine and estimate the accuracy of retrieved $n_{e}$ profiles obtained with the Abel inversion by comparing them with ionosonde data (Hajj and Romans 1998; Schreiner et al. 1999; Tsai et al. 2001). All authors obtained fractional rms differences from 20 to $40 \%$ in peak ionospheric density $(\mathrm{NmF2})$ implying differences from 10 to $20 \%$ in ionospheric critical plasma frequency ( $f \circ F 2$ ). They believe that the dominant error in the Abel inversion is due to the locally spherical symmetry assumption imposed on the ionosphere. In reality, the assumption is almost never true. Straus (1999) and Hajj et al. (2000) have presented a constrained-gradient Abel inversion method, which uses earlier simulated horizontal gradients from other ancillary data (in-situ plasma density, nadir-viewing extreme ultraviolet airglow, and vertical TEC maps derived from groundbased GPS receivers) and constrains the practical Abel inversion considering the horizontal asymmetry effect on $n_{e}$ retrieval. Both Hernandez-Pajares et al. (2000) and GarciaFernandez et al. (2003) have also presented modified Abel inversion algorithms expressing the three-dimensional retrieved $n_{e}$ as the product of a vertical TEC map and a shape function in altitude, which provided better statistics than the traditional Abel inversion assuming spherical symmetry.

In 2004, Tsai and Tsai (2004) developed a TEC compensation procedure for $n_{e}$ profiling of the GPS/MET data that also considered the effect of large-scale horizontal gradient. To assess the accuracy of the improved $n_{e}$ retrieval, validation was investigated by means of simulated GPS/ MET RO TEC data based on the Internation Reference Ionosphere (IRI) 2001 model (Bilitza 1990, 2001) and comparing the results to the "true" modeling $n_{e}$. The overestimated conditions of retrieved foF 2 between 1 to $2.5 \mathrm{MHz}$ are significantly improved from a maximum fractional mean difference of $\sim 20 \%$ to less than $10 \%$. For high $f_{o} F 2$ values, improvement of underestimations is less significant but has about $10 \%$ less than the original differences. In the following, we illustrate the data processing at National Central University in Taiwan for the ionospheric $n_{e}$ profil- ing and present preliminary results including validation checks for FS3/COSMIC ionospheric observations. We then give an overview of ionospheric $n_{e}$ profiling from GPS radio occultation observations through the Abel inversion. An iterative Abel inversion scheme using compensated TEC values is also presented and discussed. The retrieved foF 2 and $h m F 2$ results before and after improvement are examined and evaluated by comparison to an ionosonde network.

\section{THE ABEL INVERSION AND AN IMPROVED ITERATIVE SCHEME THROUGH “COMPENSATED" TECS}

A GPS-LEO occultation geometry is depicted in Fig. 1. When a GPS satellite sets or rises behind the Earth's ionosphere as seen by a LEO satellite, the GPS radio signals are received by the LEO satellite. Each occultation observation consists of a set of limb-viewing links with tangent points ranging from the LEO satellite orbit altitude to the surface of the Earth. The GPS measurements on board each FS3/COSMIC satellite are performed by a BlackJack GPS receiver (Montenbruck and Kroes 2003) developed by the Jet Propulsion laboratory, USA. GPS clock errors are removed by means of a double differencing technique. Due to the dispersive propagation properties of the ionosphere for radio signals, the limb-viewing TEC values can be derived from differential GPS phase measurements with a sampling rate of $1 \mathrm{~Hz}$ and have a resolution of 0.01 TEC units (TECU) with the BlackJack GPS receivers and when Anti-Spoofing (AS) is off. The Abel inversion technique is used under the assumptions of stationary and spherical symmetry of the local ionosphere during each occultation observation. Bending at radio frequencies in the ionosphere is less than $0.03^{\circ}$ and ray separation from straight-line propagation for observations in LEO is only about a few kilometers or less (Hajj and Romans 1998; Schreiner et al. 1999). Under the above assumptions, the "calibrated" TEC along a radio path and below the LEO orbital altitude (along the solid lines as illustrated in Fig. 1) can be determined by the limb-viewing TEC difference obtained at the occulting LEO position (P1) and another auxiliary LEO position (P2 locating at the other side of occultation and having the same ray's radial distance as P1). The calibrated TEC (or TEC') is therefore a function of tangent point's radial distance $r_{t}$ and is related to $n_{e}$ through the following integral:

$T E C^{\prime}\left(r_{t}\right)=\operatorname{TEC}(P 1)-T E C(P 2) \cong 2 \int_{r_{t}}^{r_{L} E O} \frac{r}{\sqrt{r^{2}-r_{t}^{2}}} d r$

The integral equation can be inverted by using the Abel integral transform given by Tricomi (1985) as defined by the following integral equation: 


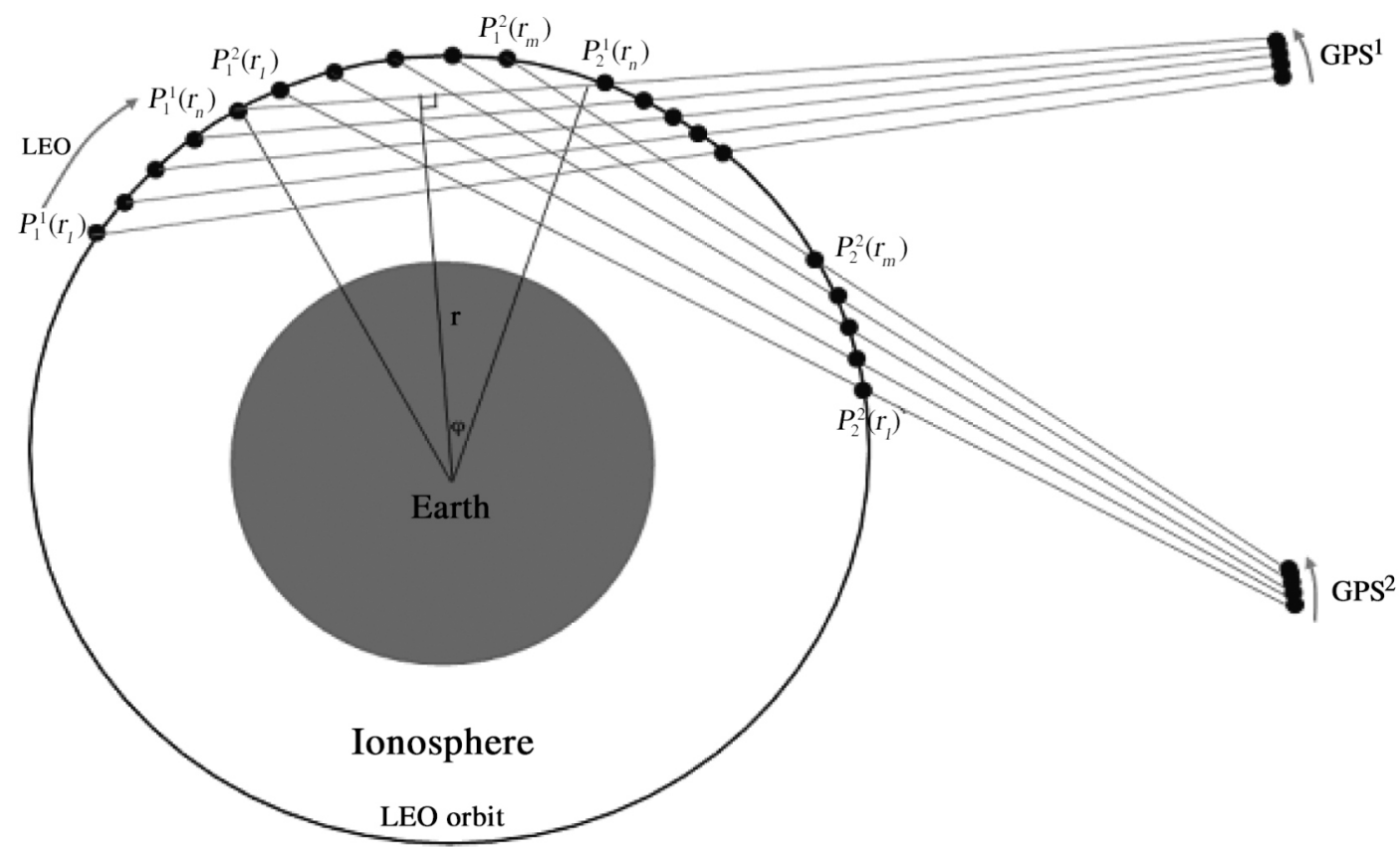

Fig. 1. Illustration of the geometry of the GPS-LEO occultation problem for ionosphere observations under the assumptions of straight-line ray propagation and co-planed LEO and GPS orbits. Arrows indicate the respective satellite's motion. $P_{l}^{i}\left(r_{k}\right)$ is the $k$ th occulting LEO position from the $i$ th GPS within a RO observation, and $P_{2}^{i}\left(r_{k}\right)$ is its corresponding calibration position. Note that this illustration is not to scale.

$n_{e}\left(r_{t}\right)=-\frac{1}{\pi} \int_{r_{t}}^{r_{L E O}} \frac{d T E C^{\prime}(r) / d r}{\sqrt{r^{2}-r_{t}^{2}}} d r$

The $n_{e}$ value at a tangent point's radial distance $r_{t}$ can then be computed in a recursive way starting from the outer rays and its $n_{e}$ profile obtained. In general, the assumption of spherical symmetry of local ionosphere, i.e., the $n_{e}$ value is a function of radial distance only, used in the Abel integral transform is not realistic. This paper proposes an improvement on the classical approach of the Abel inversion and considers horizontal gradients of $n_{e}$ distribution. These gradients are obtained from nearby occultation observations by interpolating the $n_{e}$ profiles retrieved from the standard Abel inversion. It is noted that each FS3/COSMIC spacecraft was equipped with two occultation antenna that tracked the GPS satellites in view of both setting and rising behind the Earth's atmosphere and ionosphere. The footprint of the targeted occultation may cover a wide geographic area and overlap other occultation observation areas. The retrieved $n_{e}$ from nearby occultations can then provide horizontal gradients from cubic spline fitting at specified altitudes. In the improved Abel inversion process, we define an aggregated occultation number as the number of occultation observations for which the peak position projections onto the LEO orbital plane are located within the bottom baseline projection of the targeted occultation. The left $n_{e}$ panel of Fig. 2, in this case greater than $40^{\circ}$ of LEO orbital plane angle in equatorial region, depicts nine aggregated occultations recorded on 13 July 2006, during the IOP experiment of FS3/COSMIC. The tangent point trace of the targeted occultation is presented in darker dots, and the tangent points of eight nearby occultations are presented in lighter dots. High gradients in horizontal $n_{e}$ occur in this case: the point $\mathrm{A}, \mathrm{B}$, and $\mathrm{C}$ from the nearby profiles are located at the same altitude, but their $n_{e}$ differences may reach values close to $40 \%$. In the standard Abel inversion process, the ionospheric $n_{e}$ is assumed to have local spherical symmetry and can be retrieved as a function of the radial distance only as $n_{e}(r)$ during each occultation measurement.

In this study we further define ionospheric $n_{e}$ to be expressed by a two-dimensional function $n_{e 2 d}(r, \varphi)$ of the radial distance $r$ and the LEO orbital plane angle $\varphi . n_{e 2 d}(r, \varphi)$ has been determined by the interpolation of a cubic spline fit to a set of close-up $n_{e}$ profiles, which have first been retrieved by the Abel inversion to each of the aggregated occultation observations. The calibrated TECs should be "compensated" by values of the difference between the original and spherical symmetry $n_{e}$ and the two-dimensional $n_{e}$ along the GPS-LEO occultation rays. The Abel inversion process can then be applied to the "compensated" path TECs, which are defined to be the originally determined path TECs but compensated by the line integral of the $n_{e}$ differences between $n_{e}(r)$ and $n_{e 2 d}(r, \varphi)$ in the following:

$T E C *(r)=T E C^{\prime}(r)+\int_{P 1}^{P 2^{\prime}}\left[n_{e}(r)-n_{e 2 d}(r, \varphi)\right] d l$ 

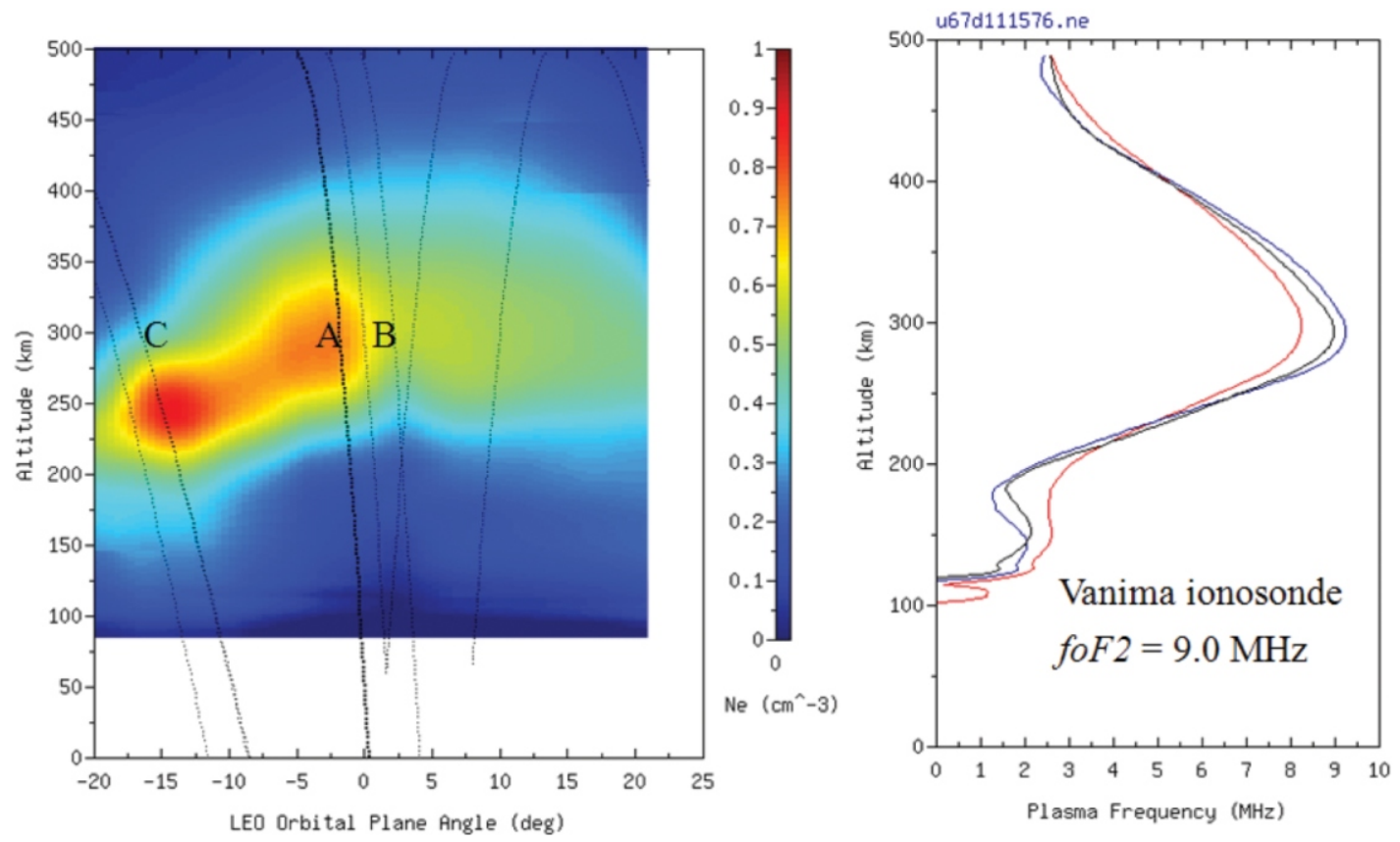

Fig. 2. An example of horizontal gradients in $n_{e}$ observed from FS3/COSMIC and the retrieved profiles using calibrated TEC (red line), compensated TEC after one iteration (blue line) and two iterations (black line). The tangent point trace of the targeted occultation is presented in darker dots, and the tangent points of eight nearby occultations are presented in lighter dots. Point $\mathrm{A}$ has the $n_{e}$ peak of the targeted profile. Points $\mathrm{B}$ and $\mathrm{C}$ from the nearby profiles have the same altitude as Point A, but their $n_{e}$ differences may reach values close to $40 \%$.

and

$n_{e} *\left(r_{t}\right)=-\frac{1}{\pi} \int_{r_{t}}^{r_{I E O}} \frac{d T E C *(r) / d r}{\sqrt{r^{2}-r_{t}^{2}}} d r$

where $\mathrm{P}^{\prime}$ ' is the corresponding calibration position of a LEO satellite position, P1, with the same radial distance along the transmitter-receiver line-of-sight. We note that $n_{e}(r)$ can be updated when the Abel inversion is applied again on compensated path TEC values but not calibrated TECs, and further aggregated $n_{e}$ profiles can be interpolated again to derive new $n_{e 2 d}(r, \varphi)$ and be used to calculate new compensated path TECs. Such procedures could be repeated to approach a reliable result. The right panel of Fig. 2 shows the original $n_{e}$ profile (in red line) using calibrated TECs and the improved profiles after one (in blue line) and two (in black line) iterations using compensated TECs. We further note that the modeled horizontal gradients are obtained by collocated $n_{e}$ profiles determined by the previous Abel inversion procedure and then should be affected by the previous "contaminated" $n_{e}$ profiles, especially for systematic errors such as in the bottom part of the profiles. This would affect the potential convergence and accuracy of the compensated TECs as well. Figure 3 shows a flowchart summarizing the improved Abel scheme through the compensated TEC values. The left side presents the standard $n_{e}$ retrieval from the Abel inversion through

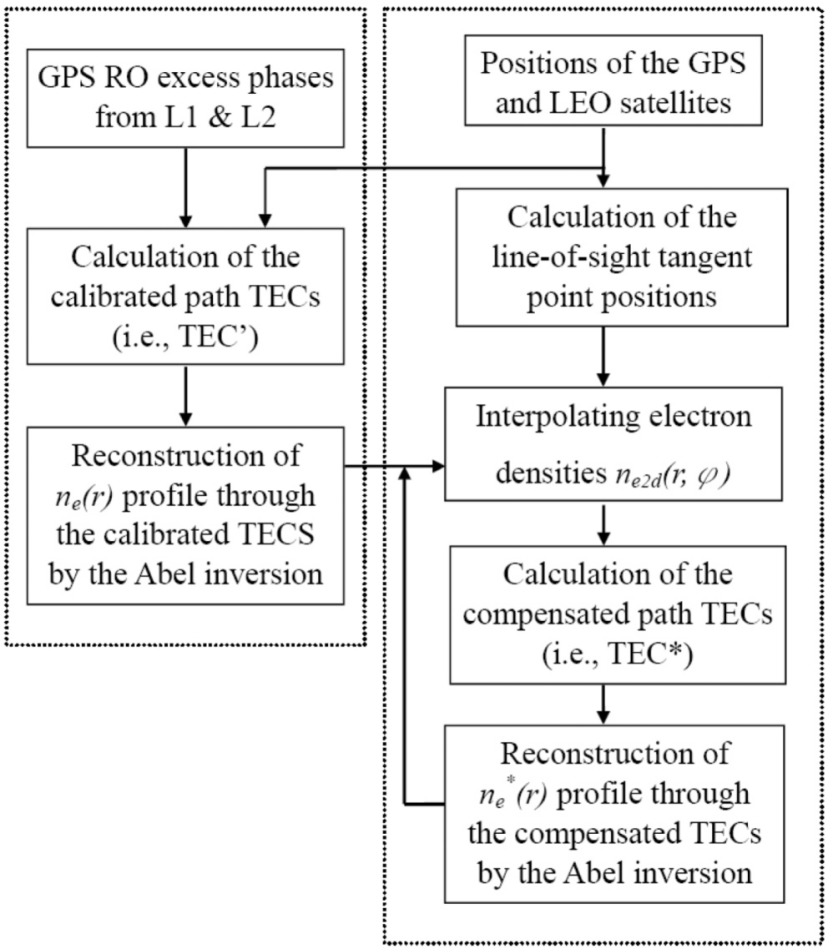

Fig. 3. A flowchart summarizing the improved Abel inversion scheme through the compensated TEC values: the left side presents the standard $n_{e}$ retrieval from the Abel inversion, and the right shows additional iterative procedures used to calculate interpolating $n_{e}$ values, the compensated TECs, and then the new $n_{e}$ retrieval from the Abel inversion again based on compensated TECs. 
calibrated path TECs. The right side shows additional and iterative procedures used to calculate interpolating $n_{e}$ values, the compensated TECs, and then the new $n_{e}$ retrieval from the Abel inversion again but based on compensated TECs.

As described the $n_{e}$ profiles retrieved from the Abel inversion have the principle averaging character in Earth angle because of the spherical symmetry assumption. Point A had a maximum $n_{e}$ at an altitude of $\sim 300 \mathrm{~km}$, thus the retrieved foF $2(8.26 \mathrm{MHz})$ from the standard Abel inversion was less than the verified value of $9.0 \mathrm{MHz}$, which was obtained independently from ionosonde measurements at Vanima, Papua New Guinea. As shown in the right panel of Fig. 2, the retrieved profiles using compensated TECs represent a successful improvement in the underestimates around the F2layer peak and provide better foF 2 values from 9.26 to $9.02 \mathrm{MHz}$ after one and two iterations, respectively.

\section{3. foF2 COMPARISONS WITH IONOSONDE MEASUREMENTS}

In order to assess the accuracy of the FS3/COSMIC $n_{e}$ retrievals, we compare the F2-layer critical frequency and peak electron density height estimates (i.e., $f_{o F}$ and $h m F 2$ ) of FS3/COSMIC RO observations with ionosonde data from a network of 49 stations at locations shown by the black solid squares in Fig. 4. Those ionosonde foF $2 \mathrm{~s}$ and $h m F 2 \mathrm{~s}$ were scaled from the sounding frequency versus virtual range ionograms and provided by the Space Physics Inter- active Data Resource (SPIDR), National Geophysical Data Center (NGDC), NOAA (http://spidr.ngdc.noaa.gov/spidr/) (O'Loughlin 1997). It has been shown that ionosonde measurements can provide accurate estimate of the critical ionospheric plasma frequency from ionogram analyses (Piggott and Rawer 1978; Paul and Mackison 1981). In contrast to ground-based ionosonde observations, the main advantages of the GPS radio occultation technique are global coverage, high vertical resolution, all-altitude capability, and the ability to observe over both ocean and land.

Figure 4 shows the geographic distribution of occultation observations during the IOP experiment (day number 171 - 270, 2006) by FS3/COSMIC. About 270000 occultation measurements were performed; vertical profiles of atmospheric parameters were derived from about 180000 occultations ( $\sim 70 \%$ of the recorded measurements). For the remaining occultation measurements the atmospheric excess phase calibration failed and the $n_{e}$ profiles could not be obtained. The failed measurements were very often caused by the locking onto the GPS carrier signals either starting too late or ending too early. A comprehensive view of the numbers of successfully retrieved $n_{e}$ profiles from RO measurements on board FS3/COSMIC is shown in Fig. 5. The number of RO measurements approaches $\sim 400$ per day and $\sim 2000$ in total from the six LEO satellites. The number of retrieved profiles during the first few weeks of the IOP experiment is low because the GPS scheduling was not optimized during that period.

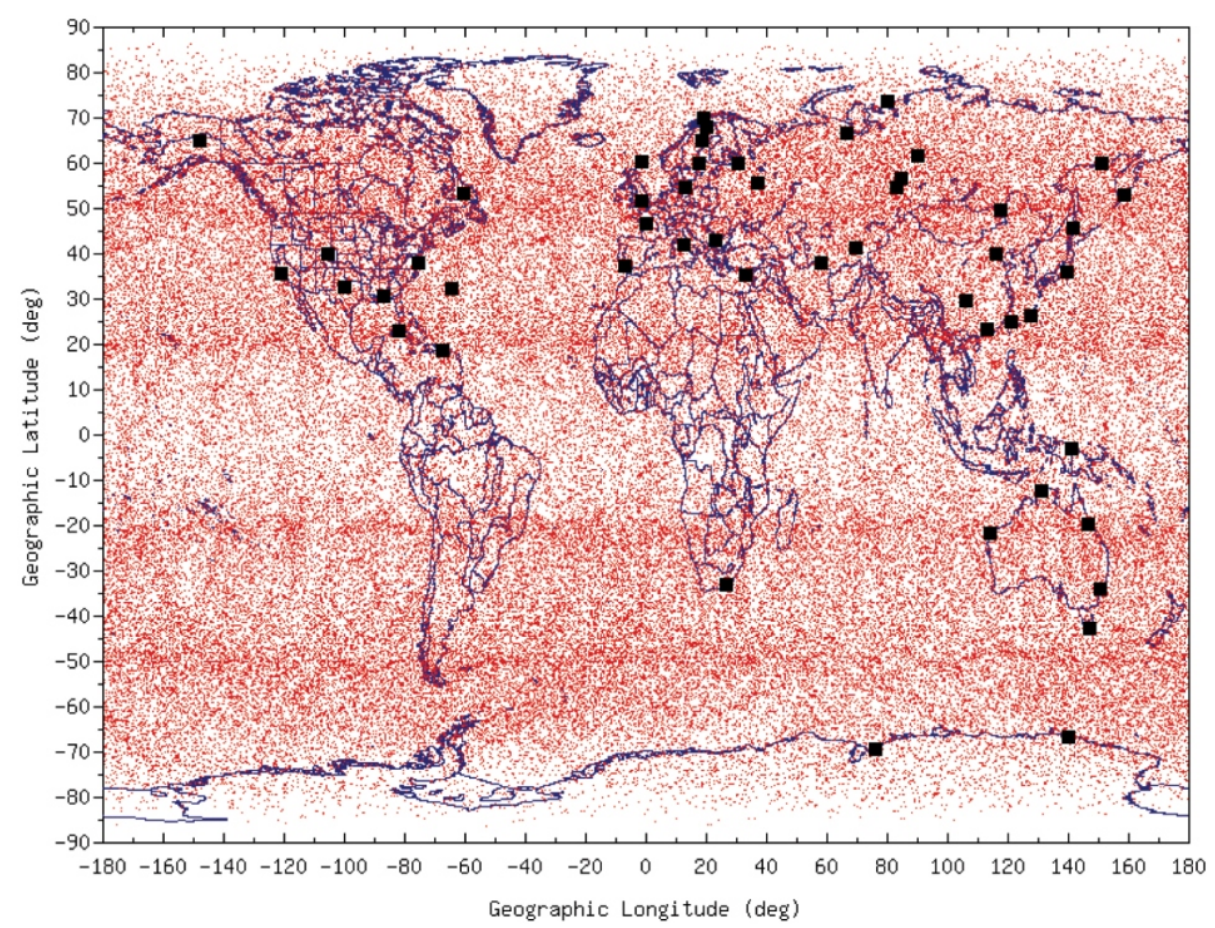

Fig. 4. Geographic distribution of 49 ionosonde sites (black solid squares) and $\sim 180000$ RO measurements (red dots) obtained by FS3/COSMIC during the IOP experiment. 


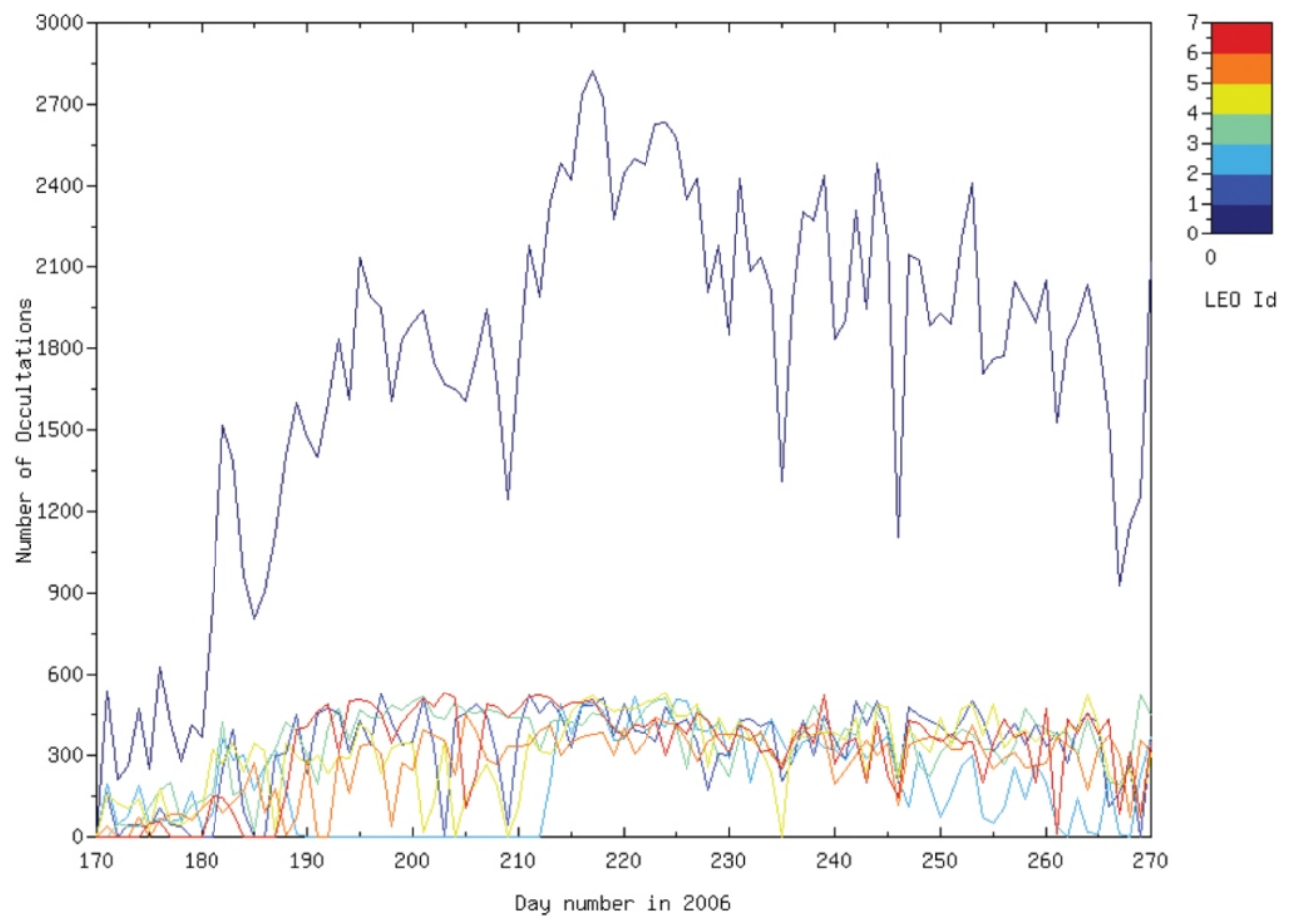

Fig. 5. Number of $n_{e}$ profiles successfully retrieved from ionospheric RO measurements on board FS3/COSMIC during the IOP experiment.

During the IOP experiment period from day number 171 - 270 in 2006, we collected $\sim 5000$ matches out of $\sim 180000$ occultations where the tangent point location of the F2 peak of each occultation was located within \pm 5 degrees of the ionosonde sites in latitude and longitude and when the digisonde measurements were within \pm 0.5 hour of each occultation observing time. Figure 6 shows a scatter plot of the FS3/COSMIC retrieved foF $2 \mathrm{~s}$ (using calibrated TECs) versus the scaled $f_{o F} 2$ from ionosonde data, their $r m s$ foF 2 difference curve in red (referred to the left $\mathrm{y}$-axis in $\mathrm{MHz}$ ), and their fractional mean $f o F 2$ difference curve in blue (referred to the right $y$-axis in percentage) as a function of the ionosonde $f_{o F}$. For $f o F 2 \mathrm{~s}$ larger than $4.5 \mathrm{MHz}$ the FS3/COSMIC retrieved $f_{o F}$ s are within a $20 \%$ fractional mean difference compared to the ionosonde $f_{0} F 2 \mathrm{~s}$. The FS3/COSMIC retrieved foF $2 \mathrm{~s}$ are biased low, i.e., they underestimate, with respect to the ionosonde $f o F 2$ values with a maximum $30 \%$ mean difference at $f_{0} F 2 s$ of $\sim 9 \mathrm{MHz}$, and the negative biases are generally increased as $f o F 2$ values from 4.5 to $9 \mathrm{MHz}$. On the other low foF 2 side, retrieved values are biased high (overestimated) with respect to the ionosonde data when $f_{o} F 2 \mathrm{~s}$

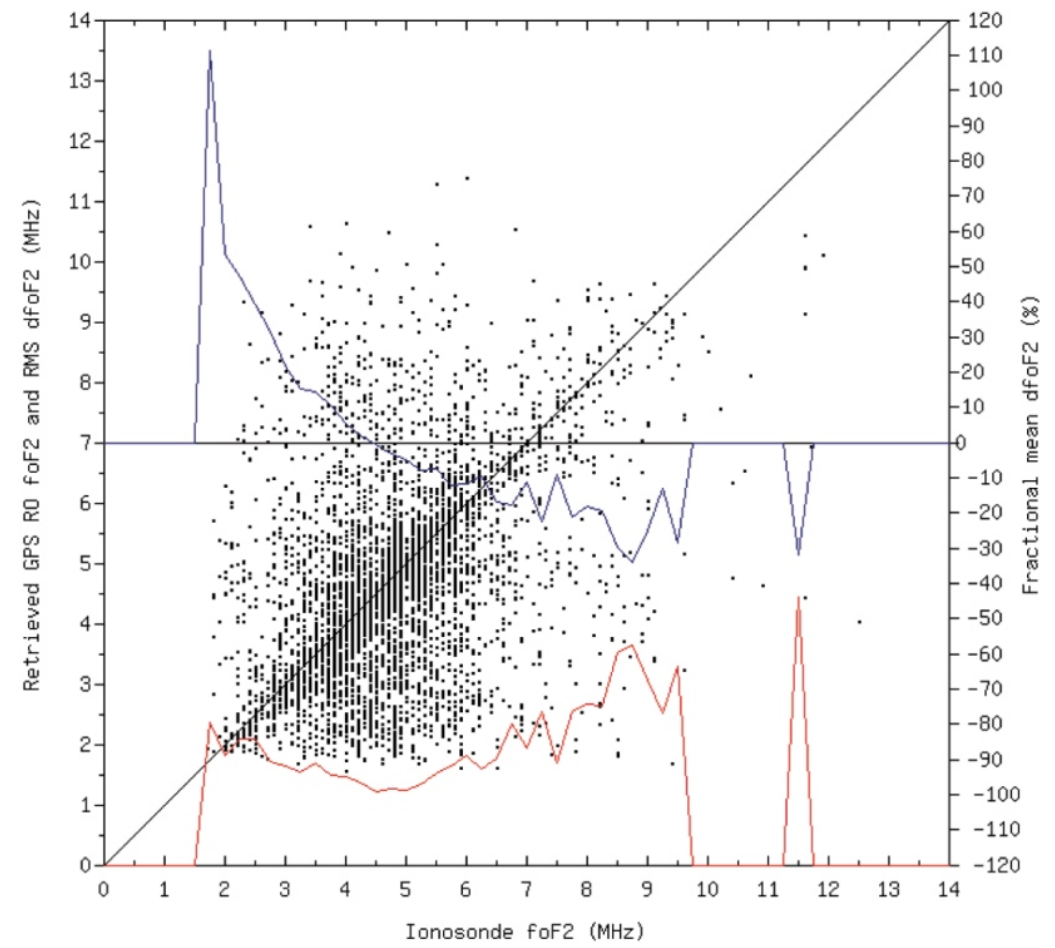

Fig. 6. A scatter plot of the FS3/COSMIC retrieved $f_{o} F 2$ s (using calibrated TECs) versus the scaled $f_{o F} 2$ from ionosonde data, their $r m s$ foF 2 difference (red, refer to the left $\mathrm{y}$-axis in $\mathrm{MHz}$ ), and their fractional mean $f o F 2$ difference (blue, refer to the right $\mathrm{y}$-axis in percentage) as a function of the ionosonde $f_{o F} 2$ during the IOP experiment. 
are less than $4 \mathrm{MHz}$. The overestimated condition can be approached to a maximum $\sim 100 \%$ mean difference for $f o F 2$ at $2 \mathrm{MHz}$, and the positive biases are also increased as $f_{o F} 2$ values from 4 to $2 \mathrm{MHz}$. The rms foF 2 difference curve in red shows results of $\sim 2 \mathrm{MHz}$ at $f o F 2$ values less than $7 \mathrm{MHz}$ but to be greater than $2 \mathrm{MHz}$ when $f_{o F} 2$ values are larger than $7 \mathrm{MHz}$. Another evaluation of the FS3/COSMIC retrieved $f_{O} F 2 \mathrm{~s}$ through the iterative scheme (after two iterations) on compensated TECs is shown in Fig. 7. Similar results of underestimated and overestimated $f_{o} F 2 \mathrm{~s}$ are obtained in high foF2 values (from 4.5 to $9 \mathrm{MHz}$ ) and low foF 2 values (from 2 to $4 \mathrm{MHz}$ ), respectively. However the fractional mean and $r m s$ foF2 differences are significantly improved. The average $r m s$ foF 2 differences are improved from 1.67 to $1.07 \mathrm{MHz}$.

In relation to GPS/MET, the FS3/COSMIC project placed six micro-satellites in LEO, and each micro-satellite has been equipped with two high-gain occultation antennas to track the GPS satellites in both views of setting and rising. Especially during the IOP experiment, the five spacecraft FM1, FM3 - FM6 were waiting for orbit rises at adjacent parking orbits of $\sim 500 \mathrm{~km}$ altitude. Quintuple RO observations rather than just single spacecraft observations were obtained at the parking orbit, and more occultation observations can be overlapped (i.e., a larger aggregated occultation number can be obtained) within a certain footprint area. During the FS3/COSMIC IOP experiment the aggregated occultation number can approach 20 or more. There could also be one aggregated occultation (the targeted occultation itself) when the targeted occultation is in view of grazing. The matched $\mathrm{RO}$ observations to ionosonde observations had a peak when the aggregated number is equal to 13 ; the distribution of matched RO observation numbers with the aggregated occultation number is shown by the black line in Fig. 8 . Figure 8 also shows the corresponding $r m s$ and mean foF 2 difference changes between the ionosonde measurements and the FS3/COSMIC retrievals under different aggregated occultation numbers and after one, two, three, and four iteration processes of the improved Abel inversion scheme. It is clearly shown that more aggregated occultations within the targeted occultation area result in less $r m s$ foF 2 difference and improve $f o F 2$ retrieval. This result is as expected because more occultations within the targeted area can be used to interpolate more accurate $n_{e}$ and then compensated path TECs. There is no typical convergence after one, two, three, and four iterations in the recursive $n_{e}$ retrieval processes, still two iterations are used for statistics comparing the ionosonde data in this paper.

\section{4. $h m F 2$ COMPARISONS WITH IONOSONDE MEASUREMENTS}

Ionosondes can provide measurements of the virtual height via radio reflections that are recorded as ionograms. There is no way to directly scale the true height $(h m F 2)$ of the maximum $n_{e}$ of the F2-layer from ionograms. For an assumption of a simple ionospheric layer, in which the variation of $n_{e}$ with height is parabolic with plasma frequency, the virtual height at a plasma frequency of 0.834 foF 2 in the absence of the Earth's magnetic field equals $h m F 2$ and can be used to estimate $h m F 2$. Other techniques for estimating $h m F 2$ can be carried out by the conversion of a plasma frequency versus virtual height curve to an $n_{e}$ true height profile, i.e., true height analyses, or determined by empirical equations with the transmission factor $M(3000) F 2$ and a correction $(\Delta M)$ to $M(3000) F 2$, formulated in terms of the ratio foF $2 / f o E$ (Dudeney 1983). As described in the previous section, we collected $\sim 5000$ matches during the IOP experiment period. However, only $40 \%$ of these ionograms have $h m F 2 \mathrm{~s}$ archived and available in the SPIDR data base. Figure 9 shows a scatter plot of the FS3/COSMIC retrieved $h m F 2$ s (using calibrated TECs) versus the scaled $h m F 2$ from ionosonde data under dots in black for latitude greater than $20^{\circ}$, in green for latitude between $20^{\circ}$ and $-20^{\circ}$ (the equatorial region), and in red for latitudes less than $-20^{\circ}$. Figure 9 also shows their $r m s ~ h m F 2$ difference curve in red (referred to the left $\mathrm{y}$-axis in $\mathrm{km}$ ) and their fractional mean $h m F 2$ difference curve in blue (referred to the right y-axis in percent-

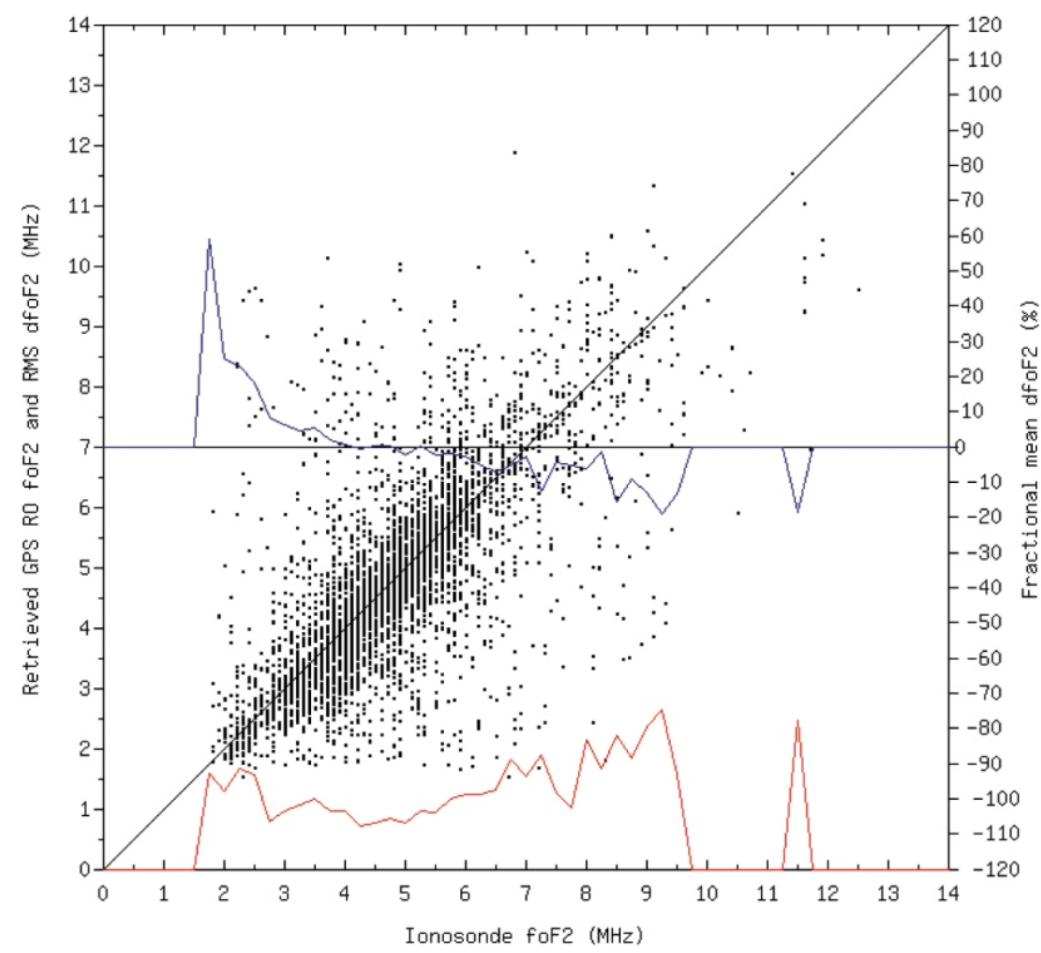

Fig. 7. As in Fig. 6 but applying to compensated TECs in RO retrieval. 


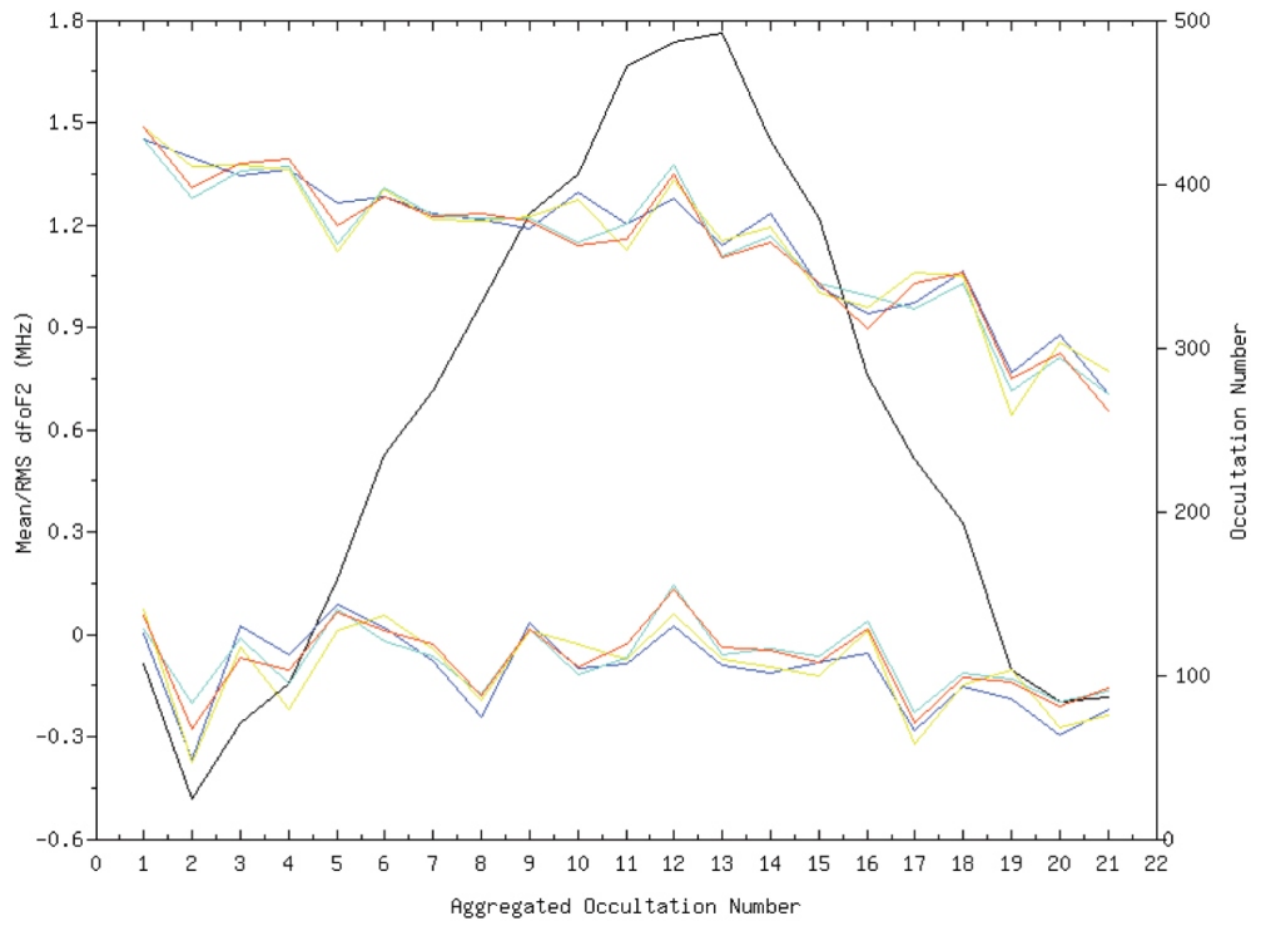

Fig. 8. The distribution of matched RO observations (refer to the right y-axis and represent in black), rms foF2 differences (the upper line set refers to the left y-axis and is in blue, yellow, cyan, and red after one, two, three, and four iteration processes, respectively), and mean $f_{0} F 2$ differences (the lower line set refers to the left y-axis) with the aggregated occultation number.

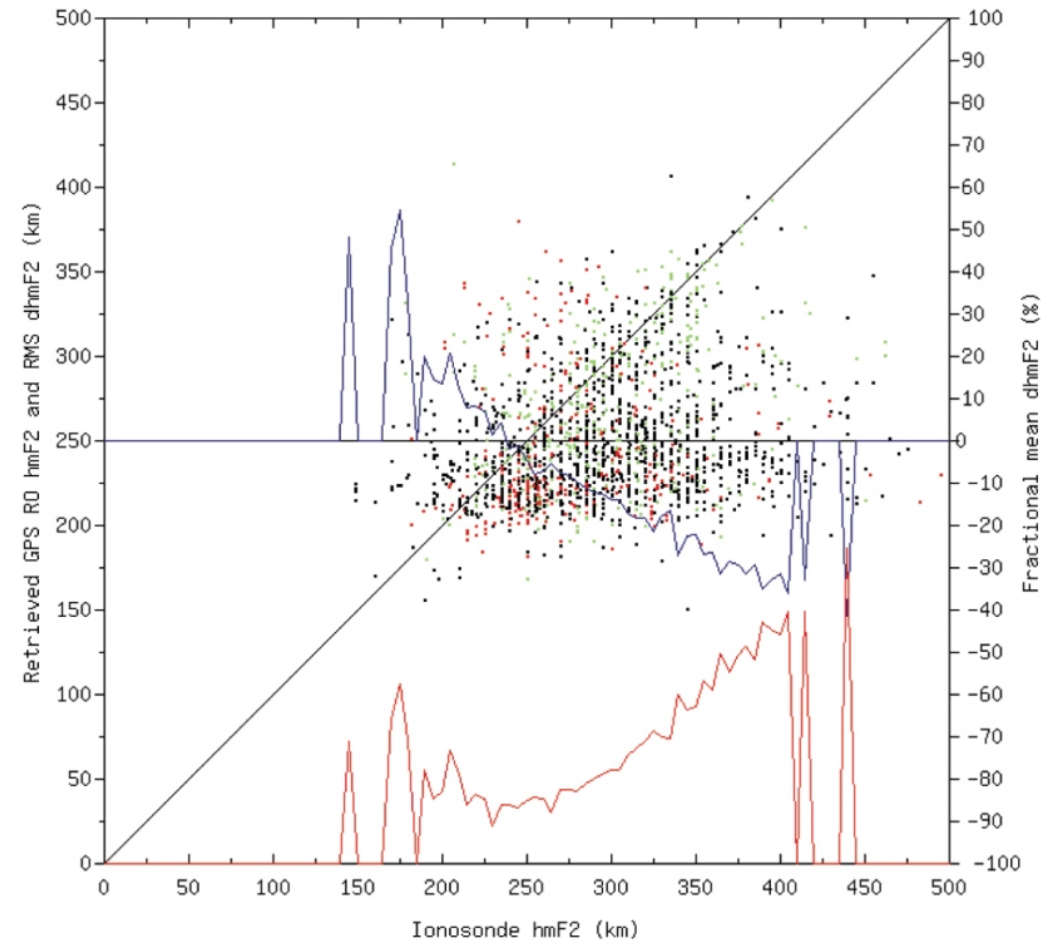

Fig. 9. A scatter plot of the FS3/COSMIC retrieved $h m F 2 s$ (using calibrated TECs) versus the scaled ionosonde $h m F 2$, their $r m s h m F 2$ difference (red, refer to the left y-axis in $\mathrm{km}$ ), and their fractional mean $h m F 2$ difference (blue, refer to the right y-axis in percentage) as a function of the ionosonde $h m F 2$ during the IOP experiment. age) as a function of the ionosonde $h m F 2$. The analysis shows that the FS3/COSMIC $h m F 2$ s do not coincide well with the ionosonde $h m F 2$ s. Another evaluation of the FS3/COSMIC retrieved $h m F 2$ s through the iterative scheme (after two iterations) on compensated TECs is shown in Fig. 10. The new FS3/COSMIC $h m F 2$ s are not coincident with the scaled ionosonde $h m F 2$ s, either. However, the scattered graphs of black dots (latitudes greater than $20^{\circ}$ ) and red dots (latitudes less than $-20^{\circ}$ ) are narrower than those in Fig. 9. Figure 11 shows zonal mean $h m F 2$ differences between the $\mathrm{FS} 3 / \mathrm{COS}$ MIC $h m F 2$ (using compensated TECs) and ionosonde $h m F 2$ for data sets within every one hour local time and $5^{\circ}$ wide latitude. The analysis shows that within the equatorial region (during both day and nighttime) and at mid and high latitudes of the Southern $\mathrm{He}-$ misphere (during nighttime only) both FS3/ COSMIC and ionosonde $h m F 2 \mathrm{~s}$ are more coincident to the other regions, and in mid and high latitudes of the Northern Hemisphere (during both day and nighttime) and in mid and high latitudes of the Southern Hemisphere (during daytime only) the FS3/ 
COSMIC $h m F 2$ s have considerable negative biases greater than $50 \mathrm{~km}$ against the ionosonde data.

It is noted that for GPS radio wave propagating in the topside ionosphere the curvature of the ray is away from the

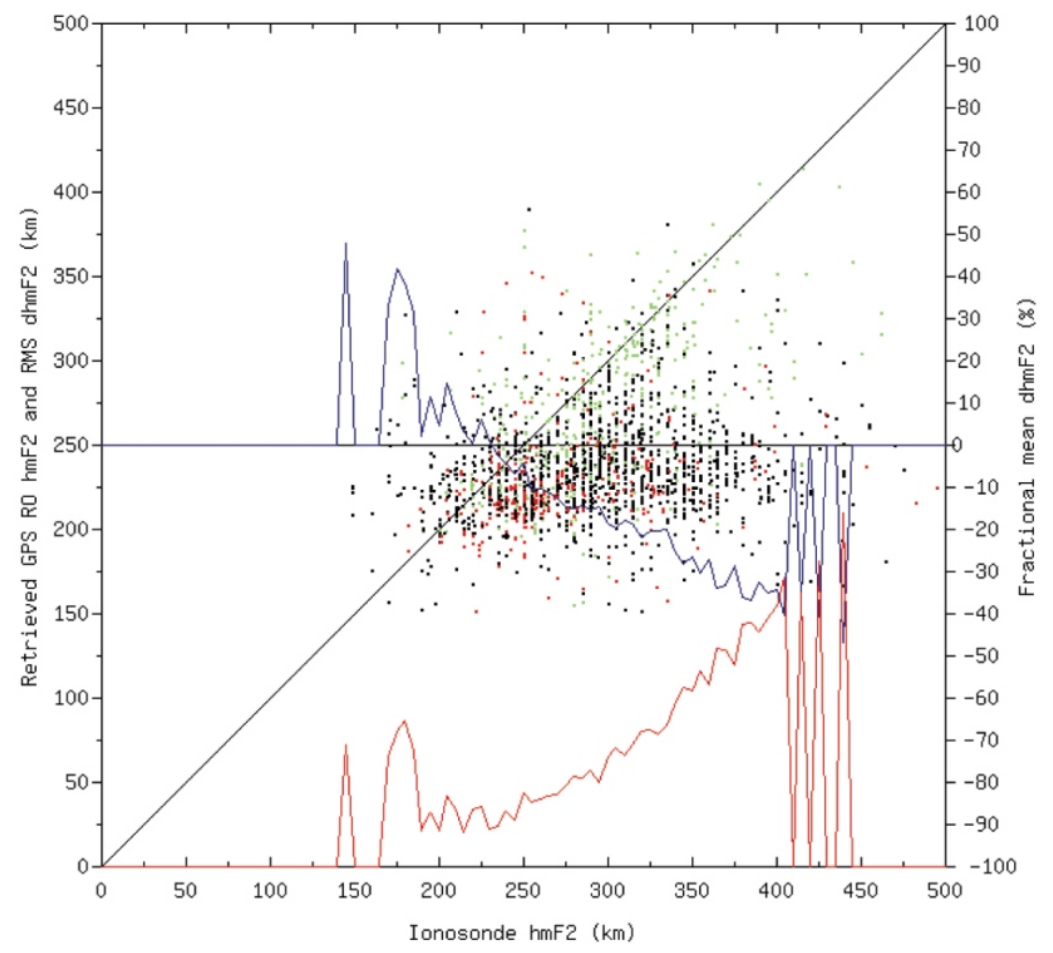

Fig. 10. As in Fig. 9 but applying to compensated TECs in RO retrieval.

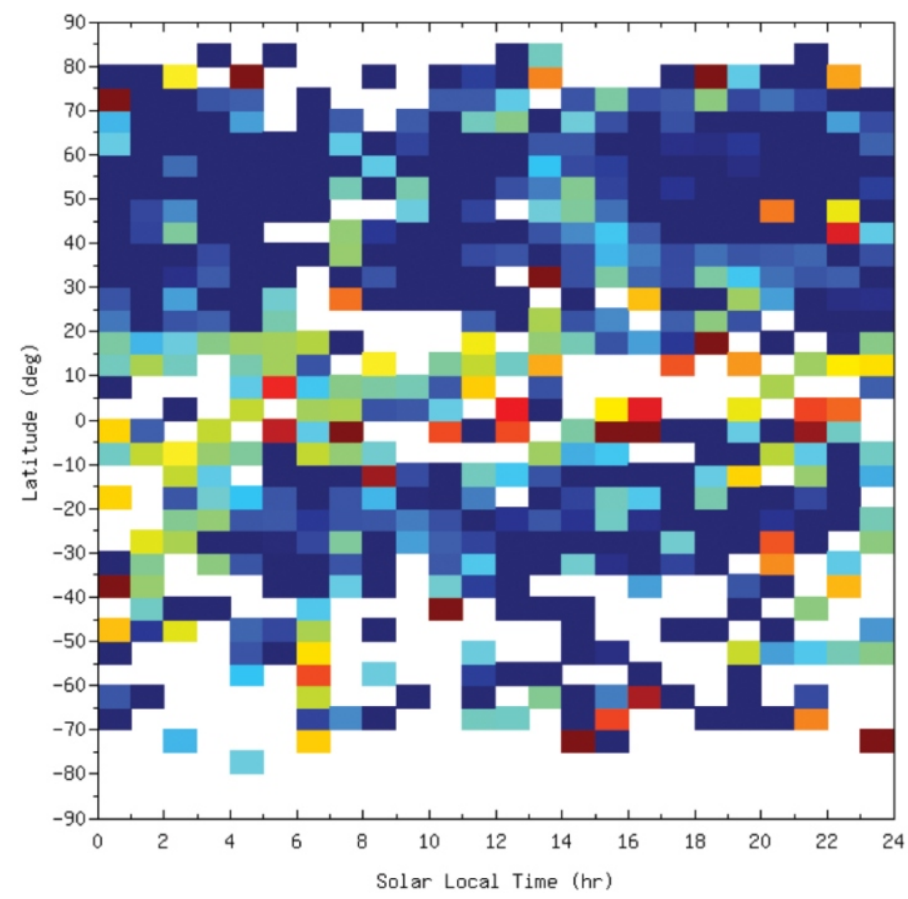

Fig. 11. Zonal means between the FS3/COSMIC peak electron density heights and the ionosonde data.

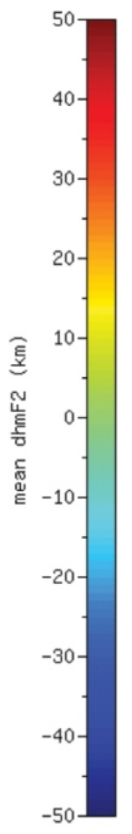

center of the earth, i.e., the bending angle is negative. In the case of a descending or setting occultation the bending of the ray is directly proportional to the amount of phase rate change and decreases from zero to a maximum negative value, and then increases to a positive value when the ray propagates through the lower atmosphere. The bending of the ray usually changes sign from negative to positive at the point where the phase delay has its maximum magnitude near the F2 peak. As described previous, the absolute magnitude of the bending angle does not exceed $0.03^{\circ}$ for both of L1 and L2 GPS frequencies in the F-region. The ray separation from straightline propagation can be estimated by $\Delta h \sim \alpha$ (in rad) $L_{R}$, where $L_{R}$ is the distance between the ray's tangent point and the LEO position and is about $3300 \mathrm{~km}$ for the FS3/COSMIC satellites at an $800-\mathrm{km}$ altitude orbit. This estimation shows that the height error of a straight-line propagation assumption is normally less than $2 \mathrm{~km}$, which is much smaller than typical vertical scales of the ionospheric $n_{e}$ and the obtained biases greater than $50 \mathrm{~km}$ described above. On the other hand, the scaled ionosonde $h m F 2 \mathrm{~s}$ could have errors attributed to: (1) an inadequate starting height by the virtual height of the lowest observed frequency and ignoring the effects of underlying ionization; (2) missing data or "gaps" in ionograms (the more available data points are, the more accurate is the retrieved $n_{e}$ profile); and (3) the poor resolution of the virtual height information (10 km for usual ionograms). Hence, we believe that the retrieved GPS RO $h m F 2$ s are more reliable than the scaled ionosonde $h m F 2$ s.

\section{SUMMARY AND DISCUSSION}

The ionospheric profiling results of the FormoSat-3/COSMIC occultation data processing during the intense observation period (from 20 June to 27 September 2006) have been presented. In this study, FS3/ COSMIC occultation data has been inverted to $n_{e}$ profiles using the Abel inversion method through two approaches of calibrated TECs and compensated TECs. The data processing technique and an improved iteration scheme to retrieve the results have been discussed. The $n_{e}$ profiles retrieved from the Abel inversion have the principle averaging 
character in Earth angle. Applying calibrated TECs directly to the Abel inversion (assuming local spherical symmetry) could induce error because of potential horizontal gradient and inhomogeneities of the ionosphere. The calibrated TECs should be "compensated" by the ray integral of the $n_{e}$ differences between the spherical symmetry $n_{e}$ and the interpolated two-dimensional $n_{e}$ from a set of close-up profiles retrieved earlier. Validation checks with independent ionospheric data from ionosonde systems show the fundamental suitability of the iteration scheme for the inversion of compensated TEC values into $n_{e}$ information. If compared with localized vertical sounding measurements, the $r m s$ foF2 differences between the ionosonde measurements and the FS3/COSMIC retrievals are improved from 1.67 to $1.07 \mathrm{MHz}$. The results also show successful improvement of overestimates (underestimates) on low (high) retrieved $f_{o F} 2 \mathrm{~s}$ and more reliable $h m F 2 \mathrm{~s}$ compared with the ionosonde data. It is further represented that more aggregated occultations within the targeted occultation area can approach better occultation $f_{o} F 2$ retrieval. It means more accurate ionospheric profiling can be achieved through more accurate compensated TEC values.

Acknowledgements This work has been supported by Projects 96-NSPO(B)-SP-FA07-02-L and NSC96-2111M008-008-012.

\section{REFERENCES}

Bilitza, D. (Ed.), 1990: International Reference Ionosphere 1990, NSSDC 90-22, Greenbelt, Maryland.

Bilitza, D., 2001: International Reference Ionosphere 2000. $R a$ dio Sci., 36, 261-275, doi: 10.1029/2000RS002432. [Link]

Dudeney, I. J., 1983: The accuracy of simple methods for determining the height of the maximum electron concentration of the F2-layer from scaled ionospheric characteristics. J. Atmos. Terr. Phys., 45, 629-640, doi: 10.1016/S00219169(83)80080-4. [Link]

Fjeldbo, G. and V. R. Eshleman, 1969: Atmosphere of Venus as studied with the Mariner $\mathrm{V}$ dual radio frequency occultation experiment. Radio Sci., 4, 879-897, doi: 10.1029/ RS004i010p00879. [Link]

Garcia-Fernandez, M., M. Hernandez-Pajares, M. Juan, and J. Sanz, 2003: Improvment of ionospheric electron density estimation with GPSMET occultations using Abel inversion and VTEC information. J. Geophys. Res., 108, 13381344, doi: 10.1029/2003JA009952. [Link]

Hajj, G. A. and L. J. Romans, 1998: Ionospheric electron density profiles obtained with the Global Positioning System: Results from the GPS/MET experiment. Radio Sci., 33, 175-190, doi: 10.1029/97RS03183. [Link]

Hajj, G. A., L. C. Lee, X. Pi, L. J. Romans, W. S. Schreiner, P. R. Straus, and C. Wang, 2000: COSMIC GPS ionospheric sensing and space weather. Terr. Atmos. Ocean. Sci., 11, 235-272.
Hernandez-Pajares, M., J. M. Juan, and J. Sanz, 2000: Improving the Abel inversion by adding ground GPS data to LEO radio occultations in the ionospheric sounding. Geophys. Res. Lett., 27, 2743-2746, doi: 10.1029/2000GL000032. [Link]

Hocke, K. and K. Igarashi, 2002: Structure of the Earth's lower ionosphere observed by GPS/MET radio occultation. $J$. Geophys. Res., 107, doi: 10.1029/2001JA900158. [Link]

Kursinski, E. R., G. A. Hajj, J. T. Schofield, R. P. Linfield, and K. R. Hardy, 1997: Observing Earth's atmosphere with radio occultation measurements using the Global Positioning System. J. Geophys. Res., 102, 23429-23465, doi: 10.1029/97JD01569. [Link]

Montenbruck, O. and R. Kroes, 2003: In-flight performance analysis of the CHAMP BlackJack Receiver. GPS Solut., 7, 74-86, doi: 10.1007/s10291-003-0055-5. [Link]

O’Loughlin, K. F., 1997: SPIDR on the web: Space physics interactive data resource on-line analysis tool. Radio Sci., 32, 2021-2026, doi: 10.1029/97RS00662. [Link]

Paul, A. K. and D. L. Mackison, 1981: Scaling of the F-layer critical frequency from digital ionograms applied to observations during the solar eclipse on 26 February 1979. J. Atmos. Terr. Phys., 43, 221-223, doi: 10.1016/00219169(81) 90042-8. [Link]

Piggott, W. R. and K. Rawer, 1978: U.R.S.I. Handbook of Ionogram Interpretation and Reduction, $2^{\text {nd }}$ Edition, World Data Center A for Solar-Terrestrial Physics, Report UAG$23 \mathrm{~A}$.

Rocken, C., R. Anthes, M. Exner, D. Hunt, S. Sokolovskiy, R. Ware, M. Gorbunov, W. Schreiner, D. Feng, B. Herman, Y. Kuo, and X. Zou, 1997: Analysis and validation of GPS/ MET data in the neutral atmosphere. J. Geophys. Res., 102, 29849-29866, doi: 10.1029/97JD02400. [Link]

Schreiner, W. S., S. V. Sokolovskiy, C. Rocken, and D. C. Hunt, 1999: Analysis and validation of GPS/MET radio occultation data in the ionosphere. Radio Sci., 34, 949-966, doi: 10.1029/1999RS900034. [Link]

Straus, P. R., 1999: Correcting GPS occultation measurements for ionospheric horizontal gradients, Proc. of Iono. Effect Symp., Alexandria, VA, June.

Tricomi, F. G., 1985: Integral Equations, Dover, Mineola, N. Y., 238 pp.

Tsai, L. C., W. H. Tsai, W. S. Schreiner, F. T. Berkey, and J. Y. Liu, 2001: Comparisons of GPS/MET retrieved ionospheric electron density and ground-based ionosonde data. Earth Planets Space, 53, 193-205.

Tsai, L. C. and W. H. Tsai, 2004: Improvement of GPS/MET ionospheric profiling and validation with Chung-Li ionosonde measurements and the IRI. Terr. Atmos. Ocean. Sci., 15, 589-607.

Ware, R., M. Exner, D. Feng, M. Gorbunov, K. Hardy, B. Herman, Y. Kuo, T. Meehan, W. Melbourne, C. Rocken, W. Schreiner, S. Sokolovskiy, F. Solheim, X. Zou, R. Anthes, S. Businger, and K. Trenberth, 1996: GPS sounding of the atmosphere from low Earth orbit: Preliminary results. Bull. 
Amer. Meteor. Soc., 77, 19-40, doi: 10.1175/1520-0477 (1996)077<0019:GSOTAF>2.0.CO;2. [Link]

Zou, X. Y., H. Kuo, and Y. R. Guo, 1995: Assimilation of atmo- spheric radio refractivity using a nonhydrostatic mesoscale model. Mon. Wea. Rev., 123, 2229-2249, doi: 10.1175/ 1520-0493(1995)123<2229:AOARRU>2.0.CO;2. [Link] 